\title{
Misdivision of Telocentrics and Isochromosomes in Wheat
}

\author{
David Kopecky ${ }^{\text {a }}$ Adam J. Lukaszewski ${ }^{\text {b }}$ \\ anstitute of Experimental Botany, Centre of the Region Haná for Biotechnological and Agricultural Research, \\ Olomouc, Czech Republic; ${ }^{b}$ Department of Botany and Plant Sciences, University of California, Riverside, CA, USA
}

\section{Keywords}

Centromere breakage · Chromosome pairing · Meiosis · Univalent

\begin{abstract}
For normal transition through meiosis, chromosomes rely on pairing with their homologues. Chromosomes which fail to pair, univalents, behave irregularly and may undergo various types of breakage across their centromeres. Here, we analyzed the meiotic behavior of misdivision products themselves: isochromosomes and telocentrics in wheat. Both types of chromosomes behaved in the same fashion as standard 2-armed chromosomes. The 2 most frequent scenarios were separation of sister chromatids in anaphase I or monopolar/bipolar attachment of the univalent to the spindle apparatus with unseparated chromatids. Misdivision was rare, and its frequency appeared directly related to the size of the centromere. The previously deduced relationship between misdivision frequency and chromosome size was likely erroneous and can be explained by a general relationship between chromosome length and the size of its centromere. Pairing of identical arms in isochromosomes did not protect them from misdivision. It is not chiasmate pairing that protects from misdivision but mechanistic issues that arise through that pairing.

(c) 2019 S. Karger AG, Basel
\end{abstract}

๑) 2019 S. Karger AG, Basel
In a typical meiotic anaphase I (AI), paired chromosomes (homologues) separate to the poles in an orderly fashion, with one member of a bivalent traveling to one pole while the other travels to the opposite pole. The fate of unpaired chromosomes, univalents, is more complicated. Often, they can lag in the division and fail to be included into daughter nuclei, and thus, are eliminated from the nuclear genome. Alternatively, a univalent lagging on the metaphase I (MI) plate may separate sister chromatids in AI followed by several different scenarios in anaphase II (AII), including misdivision of single chromatid chromosomes into separate arms [Lukaszewski, 2010]. A univalent may also be subject to misdivision in AI when pulling forces from both poles of the karyokinetic spindle tear the chromosome across the centromere. In special cases, segments of the centromere itself may become separated from the chromosome arms [Darlington, 1939; Lukaszewski, 2010]. In all cases, breakage across the centromere, misdivision, is a consequence of the absence of pairing in $\mathrm{MI}$.

The actual fate of a univalent in meiotic divisions appears to depend on the behavior of the centromeres/kinetochores of its sister chromatids and the interactions of

Both authors contributed equally to this work.

\section{KARGER}

E-Mail karger@karger.com

www.karger.com/cgr
Adam J. Lukaszewski

Department of Botany and Plant Sciences

University of California

Riverside, CA 92521 (USA)

E-Mail adam.lukaszewski@ucr.edu 
these sister centromeres with the karyokinetic spindle. In paired chromosomes, such as in bivalents and multivalents, centromeres of sister chromatids remain fused throughout the first meiotic division; they act as single units and, as a general rule, interact with only 1 pole of the karyokinetic spindle. These sister centromeres separate only prior to the second division [Dawe, 1998; Yu and Dawe, 2000; Paliulis and Nicklas, 2005]. This behavior promotes normal reduction of the chromosome number in AI and orderly separation of sister chromatids in AII. In chromosomes not involved in normal chiasmate pairing with their homologues, sister centromeres in the first division may remain fused into single units or become separated in a mitotic-like fashion. With its centromeres fused, a univalent may interact in a monopolar or a bipolar fashion with the karyokinetic spindle; the former promotes random movement of the chromosome between the poles and at times may deliver the chromosome into a daughter nucleus; the latter fixes the chromosome on the metaphase plate and may lead to separation of sister chromatids in AI, or to misdivision. The probability of sister chromatid separation in AI in univalents immobilized on the MI plate appears to be a function of time: the longer a chromosome lingers on the MI plate, the more likely it is to separate sister chromatids [Lukaszewski, 2010]. Misdivision, or breakage across the centromere, usually occurs earlier. Univalents positioned away from the MI plate do not appear to separate sister chromatids in the first meiotic division.

Telocentrics and isochromosomes in wheat are products of univalent misdivision. Most, but not all, were selected among progenies of monosomics [Sears and Sears, 1978]. Telocentrics are generally assumed to be cytologically stable; there is no systematic record of their misdivision even though Sears [1952] reported the creation of an isochromosome from telocentric 5AL. However, when present in single copy, telocentric chromosomes should be subject to misdivision in the same fashion as any univalent. Absence of the record may only reflect a technical difficulty in identification of such misdivision. On the other hand, isochromosomes are known to misdivide, and 9 telocentrics in wheat were derived via the isochromosome step [Sears and Sears, 1978].

An interesting context of centric misdivision is the possible relationship between chiasmate pairing and centromere breakage [Lukaszewski, 2013]. Chromosomes in bivalents and multivalents do not misdivide, raising the question if it is the presence of chiasmate pairing itself or mechanical issues associated with normal chiasmate pairing (such as positioning of centromeres away from the

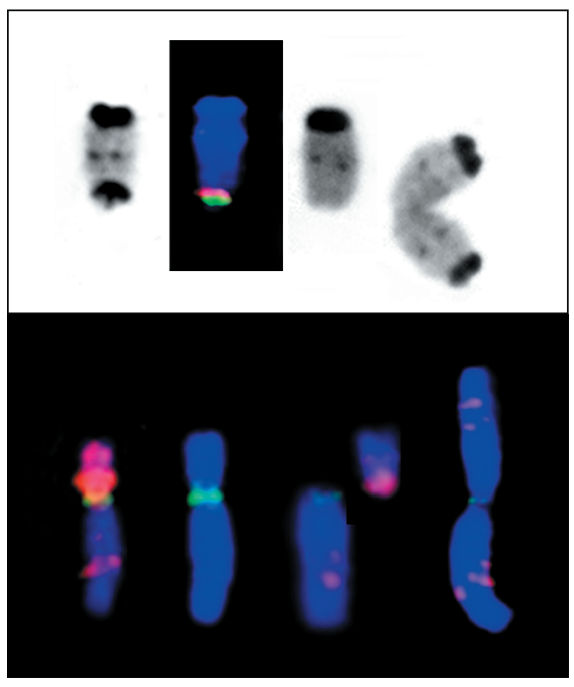

Fig. 1. Telocentrics and isochromosomes 1 RS (top) and 5B (bottom). Top, from left to right: C-banded t1RS+ (with a segment of the 1BS centromere, dark band at the bottom); t1RS+ after in situ probing with centromeric probes of wheat (green signal) and rye (red signal); t1RS after C-banding, 1BS centromeric fragment deleted; isochromosome $1 \mathrm{RS}$ without the $1 \mathrm{BS}$ centromeric fragment. Bottom, from left to right: 5B labeled with the GAA probe (red) and the wheat centromeric probe (green), counterstained with DAPI (blue). The same chromosome with only the centromeric signal (green). Telocentrics 5BL and 5BS show very little centromeric signal (green); isochromosome 5BL has the centromeric signal even smaller than $\mathrm{t}$ BBL.

metaphase plate) that protect a chromosome from misdivision. Isochromosomes offer an opportunity to discriminate between the 2 possibilities: they are composed of 2 identical arms and as such they are fully capable of chiasmate pairing. However, mechanically isochromosomes behave as univalents, as they lack independent pairing partners to properly position themselves on the metaphase plate in MI. As described by Sears [1952], isochromosomes do misdivide, but low chiasmate pairing in these reported cases does not preclude the possibility that only the non-pairing fraction contributes to misdivision. A similar case is a chromosome arm with a duplication. Reverse tandem duplication (rtd) permits chiasmate pairing in MI within a chromosome arm, but if an rtd chromosome is present in a single dose, it behaves like a univalent [Lukaszewski, 1995; Lukaszewski et al., 2012].

Misdivision of chromosomes has been recognized quite early in the history of cytogenetics [Darlington, 1939]. The process has been extensively studied in wheat [Sears, 1952], so its general features are well known. However, new techniques offer better insights into the mechanics of misdivision. This study addresses 2 specific 
Table 1. Configurations of isochromosomes in metaphase I of meiosis

\begin{tabular}{|c|c|c|c|c|c|c|c|c|}
\hline \multirow[t]{3}{*}{ Chromosome } & \multicolumn{4}{|c|}{ Arms paired, $n(\%)$} & \multicolumn{4}{|c|}{ Arms not paired, $n(\%)$} \\
\hline & \multicolumn{2}{|c|}{ attachment monopolar } & \multicolumn{2}{|c|}{ attachment bipolar } & \multicolumn{2}{|c|}{ attachment monopolar } & \multicolumn{2}{|c|}{ attachment bipolar } \\
\hline & $\begin{array}{l}\text { centromeres } \\
\text { fused }\end{array}$ & $\begin{array}{l}\text { centromeres } \\
\text { separated }\end{array}$ & $\begin{array}{l}\text { centromeres } \\
\text { fused }\end{array}$ & $\begin{array}{l}\text { centromeres } \\
\text { separated }\end{array}$ & $\begin{array}{l}\text { centromeres } \\
\text { fused }\end{array}$ & $\begin{array}{l}\text { centromeres } \\
\text { separated }\end{array}$ & $\begin{array}{l}\text { centromeres } \\
\text { fused }\end{array}$ & $\begin{array}{l}\text { centromeres } \\
\text { separated }\end{array}$ \\
\hline $\mathrm{i} 5 \mathrm{BL}^{\mathrm{a}}$ & $127(67.6)$ & & $51(27.1)$ & & $10(5.3)$ & & 0 & \\
\hline i1RS & $44(64.7)$ & $5(7.4)$ & $1(1.5)$ & $13(19.1)$ & $2(2.9)$ & $3(4.4)$ & 0 & 0 \\
\hline i6RL & $196(70.8)$ & $16(5.8)$ & $12(4.3)$ & $49(17.7)$ & $2(0.7)$ & $2(0.7)$ & 0 & 0 \\
\hline Total (rye) & $240(69.5)$ & $21(6.1)$ & $13(3.8)$ & $62(18.0)$ & $4(1.2)$ & $5(1.4)$ & 0 & 0 \\
\hline
\end{tabular}

${ }^{a}$ Very weak signal of the centromeric probe precluded reliable observation of the centromere.

questions of centric misdivision: the stability of telocentric chromosomes and isochromosomes, themselves products of misdivision, and the possible relationship between chiasmate pairing and misdivision.

\section{Materials and Methods}

\section{Plant Material}

Telocentrics and isochromosomes of rye (Secale cereale L.) chromosome arm 1RS (t1RS and i1RS, respectively) were isolated during separation of chromosome arms of centric translocations 1RS.1AL and 1RS.1BL originally from wheat (Triticum aestivum L.) cv. "Amigo" [Sebesta and Wood, 1978] and a "Veery" line, respectively [Lukaszewski, 1997a, 2017]. Among telocentrics 1RS from the Amigo source, a version was recovered with a portion of $1 \mathrm{~B}$ centromere distal to its rye centromere (Fig. 1). This chromosome was labeled t1RS+. Isochromosomes ilRS do not show the presence of a wheat segment in their centromeres. In one combination, i1RS from the Veery source was present with isochromosome i1BLrtd, where rtd indicates the presence of reverse tandem duplications at the ends of both arms. Isochromosome 6RL (i6RL) was recovered during the development of disomic single chromosome addition lines of rye chromosomes from hexaploid triticale ( $\times$ Triticosecale Wittmack) $\mathrm{cv}$. Presto to $\mathrm{cv}$. Pavon 76 [Lukaszewski, unpublished]. Telocentrics 1BL (t1BL), $5 \mathrm{BL}(\mathrm{t} 5 \mathrm{BL})$ and isochromosomes $2 \mathrm{BL}(\mathrm{i} 2 \mathrm{BL}$ ) and $5 \mathrm{BL}(\mathrm{i} 5 \mathrm{BL})$ were taken from the E.R. Sears collection of wheat aneuploids maintained by the second author. It appears that t5BL and i5BL, as they are maintained in the collection, were derived from 2 independent misdivision events of chromosome $5 \mathrm{~B}$ even though another telocentric 5BL was also produced from i5BL [Sears and Sears, 1978].

\section{In situ Hybridization}

Anthers in appropriate stages of meiosis were fixed in a mixture of 3 parts absolute alcohol:1 part glacial acetic acid at $37^{\circ} \mathrm{C}$ for a week and frozen at $-20^{\circ} \mathrm{C}$. Mitotic chromosomes $1 \mathrm{RS}$ were observed on root tip squashes prepared according to previously described protocols [Lukaszewski, 1997a, b, 2010]. Squash preparations and in situ hybridization were done following the protocol of Massoudi-Nejad et al. [2002]. Depending on the material, chromosomes were labeled in situ using 1 or 2 of 3 probes: total ge- nomic DNA of rye, the Bilby probe [Francki, 2001] specific to rye centromeres, both labeled with digoxigenin using the DIG-Nick Translation Mix and detected with anti-DIG-FITC (Roche), and/ or probe pAct6-09 from Aegilops squarrosa labeled with biotin and detected with streptavidin-Cy3 (Roche). Probe pAct6-09 hybridizes to all wheat and rye centromeres. Clone pAct6-09 was kindly provided by Dr. B. Friebe, Kansas State University, Manhattan, KS, USA. All labeling was done according to the manufacturer's instructions (Roche). Total genomic DNA of bread wheat was sheared to 200-500-bp fragments and used as a block. The probe to block ratio was about 1:150. Chromosomes were counterstained either with $1.5 \mu \mathrm{g} / \mathrm{mL}$ DAPI or with $1.5 \mu \mathrm{g} / \mathrm{mL}$ propidium iodide in Vectashield antifade solution (Vector Laboratories). Observations were made under a Zeiss Axioscope 20 equipped with epi-fluorescence, recorded with a SPOT RT Color digital camera (Diagnostic Instruments Inc.), and processed using SPOT Advanced and Adobe Photoshop v.6 software. All images were manipulated to enhance contrast and resolution, as well as to minimize background noise and were rotated for better presentation.

\section{Results}

With the exception of i2BL, all stages of meiosis necessary for this study were collected and fixed. For i2BL, no adequate number of pollen mother cells (PMCs) undergoing second division could be found, and no data for this stage are presented. Likewise, no data are given on the behavior of ilBLrtd. While on conventionally stained preparations the frequency of intra-arm pairing in MI can be scored with good accuracy [Lukaszewski, 1995] and verified by the observations of "fragment" and "bridge" configurations in AI and AII, a similar resolution was not possible using protocols employed in this study. While i1BLrtd did not appear to differ in its behavior from other isochromosomes, detailed data are fragmentary and unreliable. The chromosome is listed among the materials because it offered clear observations on the timing of centric fusions. 
Table 2. Behavior of 4 isochromosomes and 3 telocentrics in anaphase I and II of meiosis

\begin{tabular}{|c|c|c|c|c|c|c|c|}
\hline & i1RS & i2BL & i5BL & i6RL & t1RS+ & $\mathrm{t} 1 \mathrm{BL}$ & $\mathrm{t} 5 \mathrm{BL}$ \\
\hline \multicolumn{8}{|l|}{ Anaphase I } \\
\hline Bipolar, lagging & $25(12.3 \%)$ & $7(30.5 \%)$ & $27(28.4 \%)$ & $29(13.9 \%)$ & $75(23.8 \%)$ & $30(29.7 \%)$ & $28(25.9 \%)$ \\
\hline Monopolar, lagging & $28(13.8 \%)$ & $1(4.3 \%)$ & $27(28.4 \%)$ & $26(12.4 \%)$ & $59(18.7 \%)$ & $21(20.8 \%)$ & $22(20.4 \%)$ \\
\hline Sister chromatid separation & $114(56.2 \%)$ & $14(60.9 \%)$ & $36(37.9 \%)$ & $150(71.8)$ & $176(55.9 \%)$ & $50(49.5 \%)$ & $58(53.7 \%)$ \\
\hline Misdivision & $36(17.7 \%)$ & $1(4.3 \%)$ & $5(5.3 \%)$ & $4(1.9 \%)$ & $5(1.6 \%)$ & 0 & 0 \\
\hline Bipolar, lagging & $53(72.6 \%)$ & na & $40(70.2 \%)$ & $182(82.0 \%)$ & $254(64.5 \%)$ & $62(78.5)$ & $22(66.7 \%)$ \\
\hline Monopolar, lagging & $3(4.1 \%)$ & na & $2(3.5 \%)$ & $11(5.0 \%)$ & $116(29.4 \%)$ & 0 & 0 \\
\hline Sister chromatid separation ${ }^{a}$ & $17(23.3 \%)$ & na & $15(26.3 \%)$ & $26(11.7 \%)$ & $9(2.3 \%)$ & $17(21.5 \%)$ & $11(33.3 \%)$ \\
\hline Misdivision & 0 & na & 0 & $3(1.3 \%)$ & $15(3.8 \%)$ & 0 & 0 \\
\hline Total & 73 & & 57 & 222 & 394 & 79 & 33 \\
\hline
\end{tabular}

Total counts and frequencies (in parentheses) are given.

a Two-chromatid chromosomes transmitted normally to the second division.

\section{Metaphase I}

Univalent telocentrics in MI behaved in the same fashion as univalents of standard 2-armed chromosomes in the previous study [Lukaszewski, 2010], and so the frequencies of specific positions of these telocentrics were not noted. In most cases, these univalent telocentrics were positioned off the metaphase plate, caught by fixation in various positions in their migration between the poles of the division. While the actual proportions were not noted, some telocentric chromosomes showed separation of the sister chromatid centromeres.

A total of 533 MI PMCs with isochromosomes were scored. In all cases, pairing of the arms of an isochromosome was very high, ranging from $92.6 \%$ for i1RS to $98.6 \%$ for i6RL (Table 1). A single plant was studied which, in addition to i6RL, also had t6RL, both originating from the same 2 -armed chromosome 6R (data not included in Table 1). Among 67 PMCs of this plant, in 45 PMCs (67\%) pairing was between the arms of the isochromosome leaving t6RL unpaired. In 18 PMCs (27\%), one of the arms of the isochromosome was paired with the telocentric, and in 4 PMCs $(6 \%)$, there was no pairing at all. These frequencies show clear preference for pairing within the isochromosome. With random selection of a pairing partner approximately two-thirds of pairing should be between one arm of the isochromosome and the telocentric.

Apart from frequent pairing, mechanically isochromosomes behaved in a fashion typical of univalents, usually positioned off the metaphase plate. In rye isochromosomes, in a majority of cases, sister centromeres were fused into single units (74.5\%). When sister centromeres were separated, bipolar attachment to the karyokinetic spindle was 3 times more frequent (62 vs. 21 cases) than for fused centromeres. In isochromosomes with nonpaired arms, only monopolar attachment was observed even though the proportions of fused and separated sister centromeres were similar. However, because of high inter-arm pairing, the number of observations was low and may not be representative. Configurations of sister centromeres in i5BL could not be reliably observed because of a very weak centromeric signal, further reducing the number of observations.

\section{Anaphase I}

Anaphase I was the main focus of this study, and a total of 1,054 PMCs were scored. Again, the general pattern of behavior of telocentrics and isochromosomes in AI (Table 2) was similar to that of univalents of standard 2-armed chromosomes [Lukaszewski, 2010]. In most PMCs, univalent telocentrics and isochromosomeslagged behind other chromosomes and either separated sister chromatids, or migrated intact toward one of the poles. Similarly to univalent 2-armed chromosomes [Lukaszewski, 2010], the frequency of sister chromatid separation also appeared to be related to the time spent on the metaphase plate. In PMCs in late AI, as judged by the position and appearance of other chromosomes, the frequency of sister chromatid separation was much higher than in early AI.

With low frequency, separated chromatids remained in the vicinity of the metaphase plate with no evidence of attachment to the spindle apparatus and hence, no poten- 

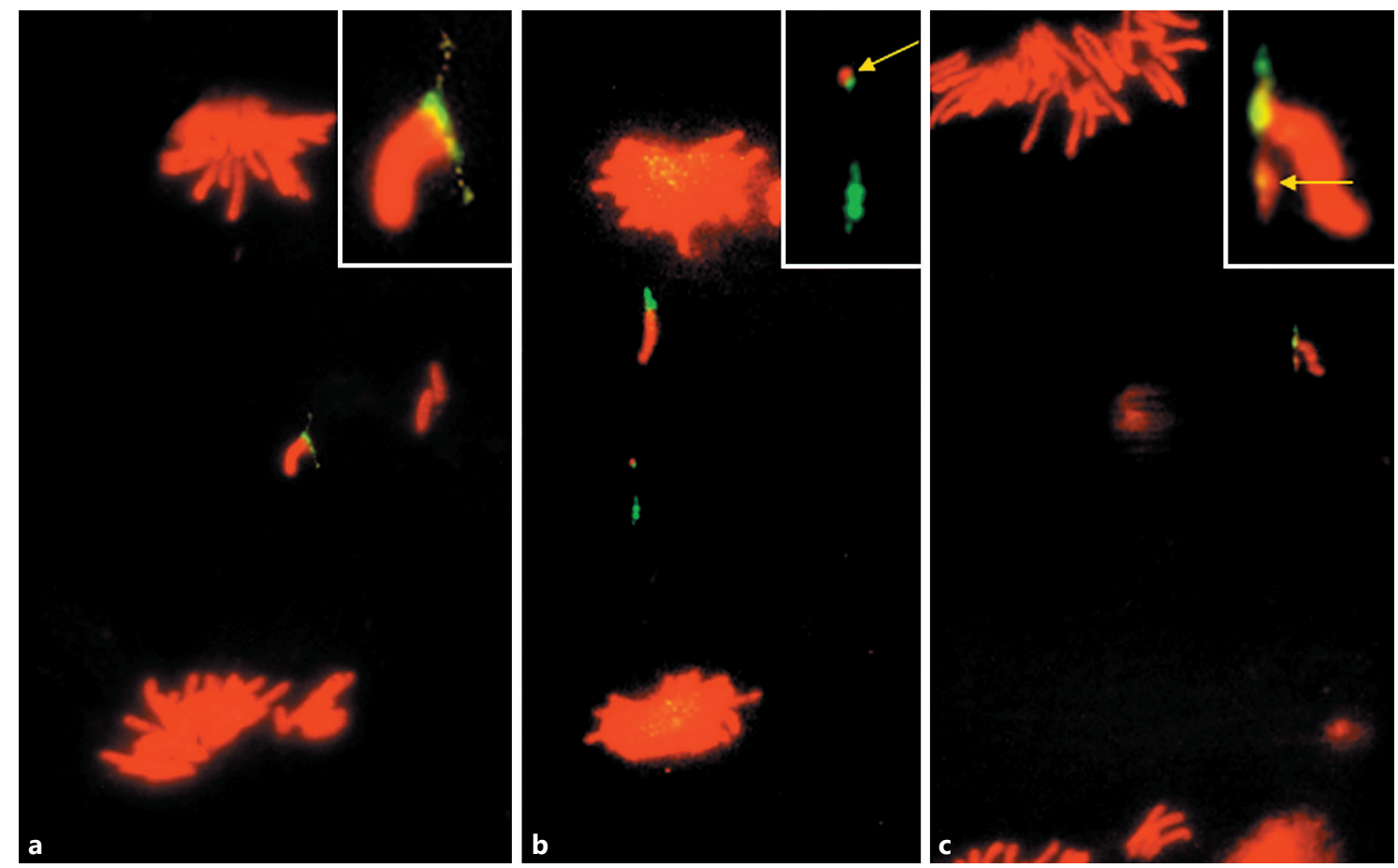

Fig. 2. Misdivision of telocentric t1RS+ in anaphase I (AI) and II (AII) of meiosis. a Bipolar attachment of t1RS+ in late AI. b Separation of a centromeric fragment from t1RS+ in AII (wheat segment of the centromeric fragment is red, arrowed). c Bipolar attachment of t1RS+ in AII; wheat centromeric fragment with a segment of $1 \mathrm{RS}$ centromere is being separated from the rest of the 1RS centromere. Green signal, rye centromere. Insets enlarge the critical details in each figure.

tial for movement to the poles. Misdivision was infrequent (Table 2), especially among telocentrics. Chromosome t1RS+, with its double-sized centromere, was the only misdividing telocentric chromosome during AI in this study, and here misdivision also included fragmentation of the centromere (Fig. 2). In isochromosomes, misdivision was more frequent (from $1.9 \%$ in i6RL to $17.7 \%$ in i1RS) and included (1) separation of arms in one of the sister chromatids (Fig. 3), (2) separation of all 4 arms of sister chromatids, and (3) separation of a sister chromatid of one of the arms from the rest of the chromosome. Misdivision of an isochromosome producing 4 single chromatids was observed 3 times among 1,054 PMCs scored.

\section{Anaphase II}

A total of 858 observations of AII PMCs were made. However, the fixation and squashing method used in this study frequently leads to separation of dyads (halves of tetrads), and in most cases only such half-tetrads were scored, and exclusively those with the centromeric signal present. Consequently, numbers presented in Table 2 overestimate the actual frequencies of events, but relative proportions of different scenarios in AII should be correct.

In cases where both sister chromatids were present in an AII cell, the division appeared to proceed normally; that is, sister chromatids separated and moved to daughter nuclei in synchrony with the remaining chromosomes. On the other hand, single chromatid chromosomes most often lagged on the metaphase plate or in its vicinity, probably as a consequence of bipolar attachment to the karyokinetic spindle. Only 18 cases of misdivision were observed, 3 for $\mathrm{i} 6 \mathrm{RL}$ and 15 for t1RS+. In the latter, misdivision involved fragmentation of the centromere. Interestingly, in 3 cases, all in MII from plants with univalent i1RS and i1BLrtd, fusion of broken centromeres was observed creating centric wheat-rye translocations (Fig. 4).

Telocentric 1RS+ with its hybrid rye/wheat centromere offered additional insights. Among 394 scored AII PMCs, 5 chromosomes had no detectable wheat centromere segment indicating centromere breakage during AI. This corresponds roughly to the misdivision frequency observed in AI (Table 2) but was lower than the frequen- 

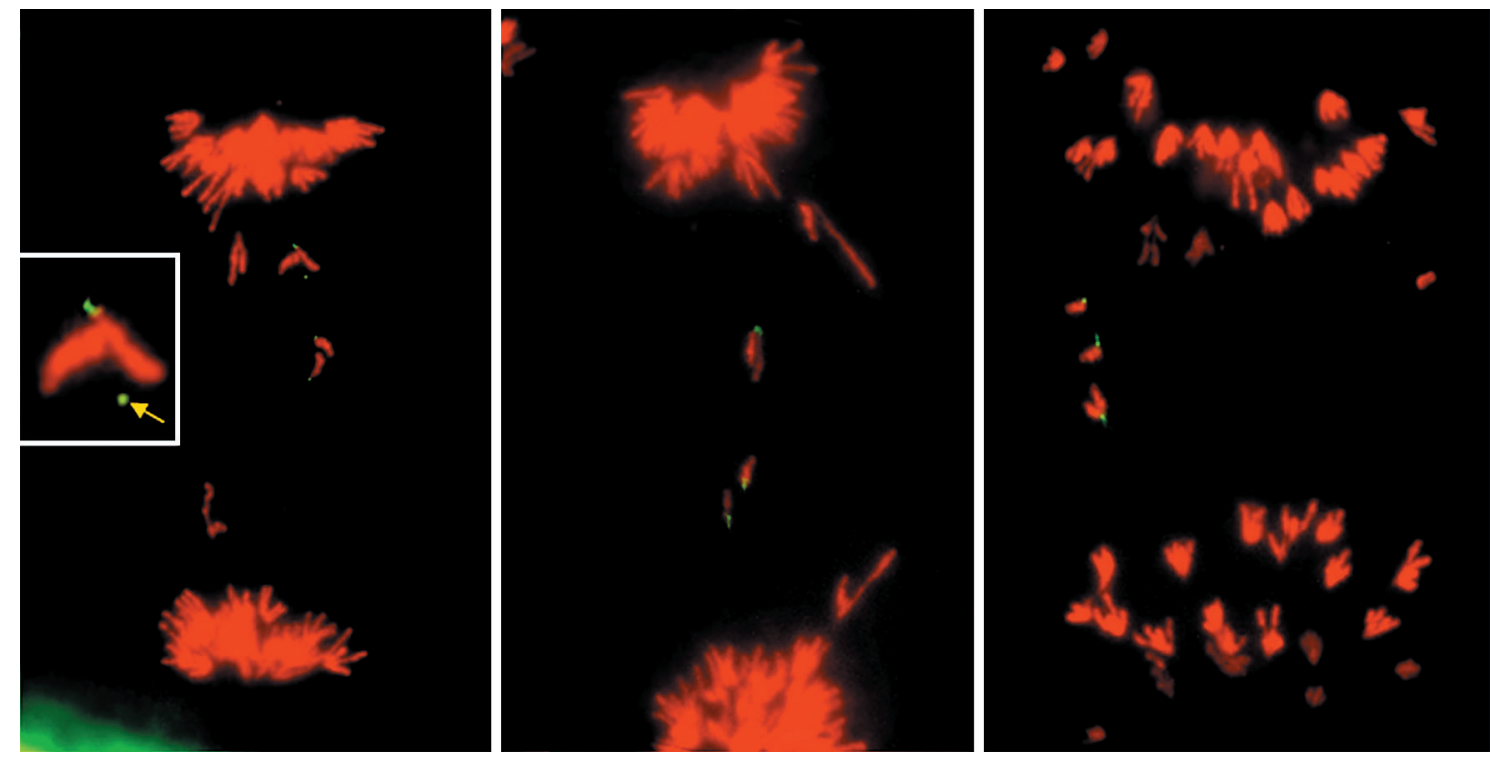

Fig. 3. Misdivision of ilRS in anaphase I of meiosis. Green signal, rye centromere. Each segment shows separation of the arms of one sister chromatid. On the left, also separation of a free centromeric fragment (inset, arrow).

cy of such chromosomes recovered among the progeny $(5$ telocentrics and 2 isochromosomes 1RS without wheat centromeric segment among $25 \mathrm{t} 1 \mathrm{RS} / \mathrm{i} 1 \mathrm{RS}$ recovered in 58 progeny, detailed data not shown).

\section{Discussion}

Centric misdivision of univalents can serve as a useful tool in plant cytogenetics. Complete sets of telocentrics and isochromosomes were produced by this mechanism [Sears, 1952; Sears and Sears, 1978], and complete genomes of related species were introgressed into wheat in the form of centric translocations [Liu et al., 2011]. The 1RS.1BL centric translocation is the most successful example of the introgression of agriculturally important traits from a close relative (rye, in this case) for improvement of wheat cultivars. About one-third of all wheat cultivars grown worldwide carry this translocation [Wang et al., 2017].

Apart from germplasm enhancement, centric misdivision itself and its products can be utilized to answer general biological questions. In this study, we used telocentrics and isochromosomes to investigate basic mechanisms of meiotic division, such as correlation of chiasmate pairing with chromosome stability and the transmission into the next generations. Our results do not offer any support to the hypothesis that chiasmate pairing protects a chromosome from misdivision. Direct observation of a
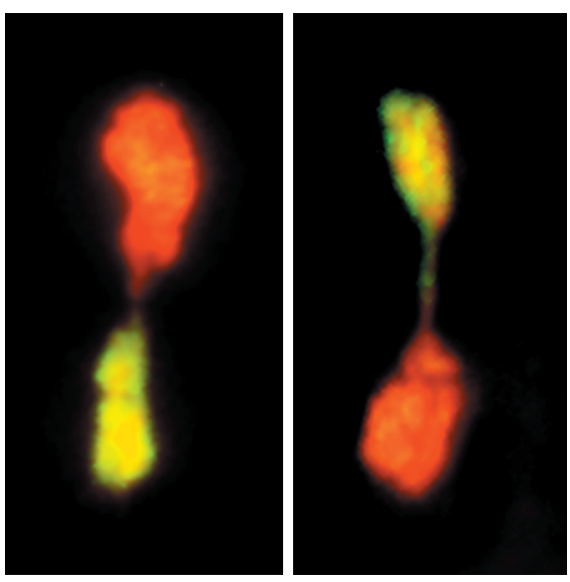

Fig. 4. Metaphase II of meiosis. Fused telocentric chromosomes produced by misdivision in AI. On the left, it appears that only 1 of 2 chromatids from each chromosome is fused. Rye chromosome arms are yellow/green; wheat arms are red.

relationship between chiasmate pairing of isochromosomes and their tendency to misdivide cannot be made because misdivision occurs in AI, after chiasmata are released. However, indirect evidence points to absence of such a relationship. Pairing frequencies of identical arms of isochromosomes in this study were very high, much higher than those reported by Sears [1952], and yet misdivision was observed. Similarly to univalents of standard 
Fig. 5. Isochromosomes 1RS (left; arrowed) and 6RL (right) each forming a ring in metaphase I. Note separated centromeric signals (green) of 6RL.
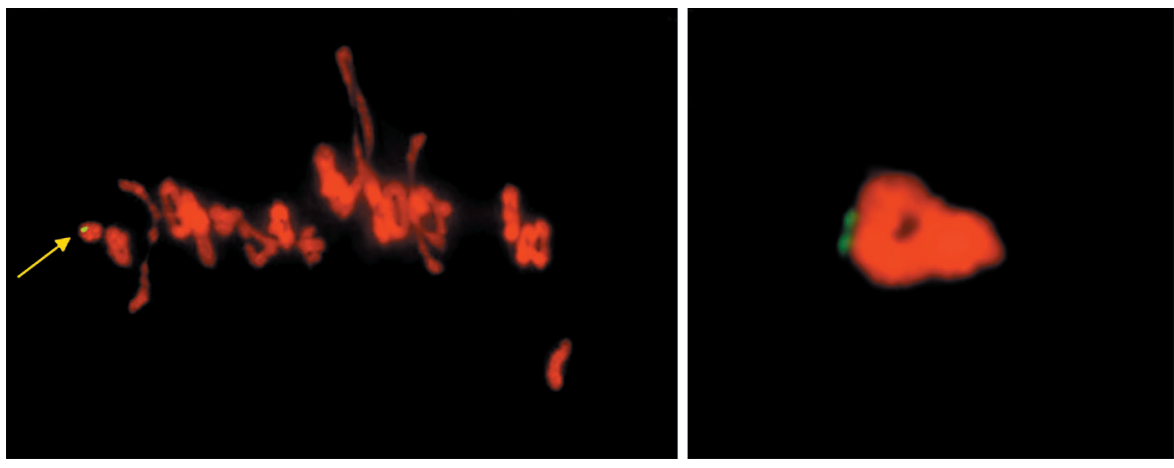

wheat chromosomes, isochromosomes were often (ca. $25 \%$ ) found in MI with separated sister centromeres, and in almost all cases those were ring univalents (Fig. 5). Therefore, it is the erratic behavior of univalents, with their tendency to bipolar attachment to the karyokinetic spindle which makes them prone to misdivision. As in the case of regular 2-armed univalents, lagging on the MI plate also increases the frequency of sister chromatid separation in $\mathrm{AI}$, and this in turn increases the chances of misdivision in AII.

In the first exercises with centric misdivision in wheat, Lukaszewski [1993, 1997a] observed that longer chromosomes, including centric translocations, misdivided more frequently than shorter chromosomes. These differences are probably a consequence of different numbers of active centromere units present, and not the chromosome length as such. The argument is advanced here that the frequency of misdivision of an individual chromosome is related to the size of its functional centromere. Further studies are needed to quantify this relationship as at present it is based only on cursory observations of centromeric signals in various chromosomes and behavior of various chromosomes when present as univalents in meiosis. It needs to be pointed out clearly that the probes used to assess centromere sizes are DNA clones/sequences but the apparatus directly responsible for misdivision is the kinetochore. The kinetochore, and not chromatin itself, interacts with the karyokinetic spindle. The length of the centromere per se, in the sense of the number of centromere-specific DNA repeats present, may actually be of little importance; the length of the CENH3 chromatin may be far more important for the stability of chromosomes [Koo et al., 2015; Guo et al., 2016]. However, it was pointed out previously [Lukaszewski, 2010] that the only part of a chromosome interacting with the karyokinetic spindle is the one labeled by the centromeric DNA probe, as those used here. In this sense, the size of the centro- meric signal generated by the DNA probes employed here is probably a good proxy for the centromere size.

A general relationship between centromere size and chromosome length may exist, but it appears quite likely that the allele-like variation also exists and is responsible for large differences in misdivision frequencies among homologues from different sources. Sears [1973] observed a 2 -fold difference in misdivision frequencies of chromosomes $3 \mathrm{~B}$ from 5 different sources but in the same genetic background; several telocentrics in wheat had to be imported into cv. Chinese Spring from other sources because they could not be generated from chromosomes of Chinese Spring [Sears and Sears, 1978]. In rice, centromere sizes detected by specific DNA probes [Cheng et al., 2002] showed a dramatic range, from almost non-detectable cytologically to occupying substantial portions of metaphase chromosomes. A similar study is yet to be conducted in wheat but DNA sequence assemblies show large differences in centromere sizes among individual chromosomes of standard cultivar Chinese Spring [International Wheat Genome Sequencing Consortium, 2018]. Centromeric repeats are rapidly evolving sequences, potentially generating variation in copy numbers [Henikoff et al., 2001]. Moreover, large variation in centromere structure including loss or amplification of centromerespecific DNA repeats and formation of dicentric chromosomes can be initiated by interspecific hybridization [Guo et al., 2016]. Such allele-like differences in the centromere structure may explain major difficulties in the production of telocentrics from some chromosomes of specific accessions of wheat while the same chromosomes from different accessions misdivide with reasonable frequencies.

Here, both the telocentric and the isochromosome of $5 \mathrm{~B}, \mathrm{t} 5 \mathrm{BL}$ and $\mathrm{i} 5 \mathrm{BL}$, show much smaller centromeric signals than the original $5 \mathrm{~B}$ from which they were derived. As a matter of fact, centromeric signals in $\mathrm{t} 5 \mathrm{BL}$ and $\mathrm{i} 5 \mathrm{BL}$ were so weak that they were rarely detectable in meiosis. 
These 2 chromosomes were not observed to misdivide in reasonably large samples. On the other hand, telocentric chromosome t1RS+, with its native centromere considerably enlarged by the addition of a portion of the centromere from $1 \mathrm{BL}$, misdivided very frequently, forming t1RS and ilRS without the wheat centromere segment and, perhaps, even with parts of its own centromere missing. Separation of centromere fragments from this t1RS+ was observed (Fig. 2) but, as in the previous study [Lukaszewski, 2010], such fragments were not recovered among the progeny. Centromere sizes in $\mathrm{t} 5 \mathrm{BL}$ and $\mathrm{i} 5 \mathrm{BL}$ are perfectly sufficient for normal somatic stability and the transmission through meiosis but appear too low for sufficiently frequent bipolar attachment to the karyokinetic spindle in meiotic divisions to generate misdivision. In maize, the centromere size, as detected by in situ probing, did not have an effect on meiotic segregation of homologues with large differences in the amounts of centromeric DNA present [Han et al., 2018].

Telocentric chromosomes are generally believed to be stable, in the sense that they do not misdivide further. However, Sears [1952] in his early observations of misdivision in wheat did report on the formation of an isochromosome from telocentric 5AL. Such an isochromosome can only be formed by misdivision, most likely in AI, followed by fusion of the centromeres of sister chromatids. Given the repetitive nature of the centromere (as illustrated by its ability to undergo consecutive misdivisions), there is no reason to assume that telocentrics would not be subject to misdivision. Moreover, one could reasonably expect large differences in susceptibility to misdivision among different telocentrics based on the length of their centromeres. Centromeres in higher organisms are composed of many units capable of interaction with the karyokinetic spindle. Bipolar attachment of any given centromere, either in MI or MII, may lead to asymmetrical division across the centromere (misdivision) with each separated arm carrying different numbers of centromeric units. This was shown in centric translocations recovered after one or more rounds of centric misdivision and fusion [Zhang et al., 2001; Wang et al., 2017] where the breakage points were spread over the entire (cytologically detectable) lengths of centromeres, in some cases leading to what appeared as almost complete replacement of the centromere of one chromosome with the centromere of another [Wang et al., 2017].

The likely relationship between centromere size and the susceptibility to misdivision is also supported by ever dropping frequencies of centric misdivision in consecutive rounds, to the point that after the third round, misdivision products were almost impossible to recover or maintain [Lukaszewski, 1997b]. Similarly, in maize, consecutive rounds of misdivision of the same centromeres came about with much reduced frequencies [Kaszas and Birchler, 1996; Phelps-Durr and Birchler, 2004; Birchler and Han, 2009]. Reduction in the size of centromeres in maize (by consecutive misdivisions) did not impact their performance in mitosis but in some cases did so in meiosis [Birchler and Han, 2013]. In this study, telocentric chromosome t1RS+ with its centromere enlarged by a substantial portion of the centromere from wheat chromosome $1 \mathrm{~B}$ was particularly prone to misdivision, readily forming isochromosomes and new telocentrics with, as far as it could be ascertained on C-banded preparations, deleted portions of the wheat centromere. Clear separations of centromeric fragments from the rest of t1RS+ chromosome were observed several times in this study (Fig. 2). On the other hand, t5BL, with a very weak hybridization signal of the centromeric probe (Fig. 1), much smaller than the one observed in a normal $5 \mathrm{~B}$ chromosome from which it originated, did not undergo misdivision to any appreciable extent. Based on these observations, we feel confident to postulate that the misdivision frequency of individual chromosomes in wheat remains in direct proportion to the size of the centromeres: small centromeres are less likely to be involved in amphitelic attachment to the karyokinetic spindle than chromosomes with larger centromeres, and hence, undergo misdivision less frequently.

The timing of fusion of broken centromeres still remains somewhat obscure. It is generally believed that standard DNA repair mechanisms, such as nonhomologous end-joining [Wyman and Kanaar, 2006] which are likely responsible for fusions of broken centromeres creating isochromosomes or centric translocations, are inactive in meiosis. Double-strand breaks created for the purpose of crossing over are repaired by the end of pachytene and so are externally induced double-stand breaks [Coogan and Rosenblum, 1988]. Lukaszewski [2010] observed numerous cases where 2 broken centromeres were present in a nucleus of a dyad, or in the second meiotic division, but no fused chromosome arms were detectable. Friebe et al. [2005], however, documented the presence of fused chromosome arms in ana-/telophase II. Similarly here, in a rather small sample of MII PMCs from plants double monosomic for i1RS and i1BLrtd, 3 instances of fused chromosome arms were observed. In one of these 3 cases, only 1 of 2 sister chromatids appear fused (Fig. 4). This establishes that fusion of broken centromeres can take place almost immediately after misdivision in AI and indicates that double-strand break repair mechanisms must function in later stages of meiosis. However, consid- 
ering the low frequency of such observations as opposed to high frequency of centric fusion products among progeny, they obviously are not very efficient at this point, and the bulk of fusion occurs post-meiotically.

This study demonstrates that telocentrics and isochromosomes, when present as univalents, are susceptible to misdivision in the same fashion as normal 2 -armed chromosomes. Their perceived high stability is probably only a consequence of reduced centromere sizes and, therefore, much reduced tendency to a bipolar attachment to the karyokinetic spindle.

\section{Disclosure Statement}

The authors have no conflicts of interest to declare.

\section{Funding Sources}

This study was done with support from the National Institute of Food and Agriculture (NIFA) Project CA-R-BPS-5411-H to A.J.L. and from the National Program of Sustainability I, grant award LO1204 to D.K.

\section{References}

Birchler JA, Han F: Maize centromeres: structure, function and epigenetics. Annu Rev Genet 43: 287-303 (2009).

- Birchler JA, Han F: Meiotic behavior of small chromosomes in maize. Front Plant Sci 4:505 (2013).

-Cheng Z, Dong F, Langdon T, Ouyang S, Buell $\mathrm{CR}$, et al: Functional rice centromeres are marked by a satellite repeat and a centromerespecific retrotransposon. Plant Cell 14:16911704 (2002).

- Coogan TP, Rosenblum IY: DNA double-strand damage and repair following $\gamma$-irradiation in isolated spermatogenic cells. Mutat Res 194: 183-191 (1988).

Darlington CD: Misdivision and the genetics of the centromere. J Genet 37:341-364 (1939).

Dawe RK: Meiotic chromosome organization and segregation in plants. Annu Rev Plant Physiol Plant Mol Biol 49:371-395 (1998).

Francki MG: Identification of Bilby, a diverged centromeric Ty1-copia retrotransposon family from cereal rye (Secale cereale L.). Genome 44:266-274 (2001).

-Friebe B, Zhang P, Linc G, Gill BS: Robertsonian translocations in wheat arise by centric misdivision of univalents at anaphase I and rejoining of broken centromeres during interkinesis of meiosis II. Cytogenet Genome Res 109: 293-297 (2005).

Guo X, Su H, Shi Q, Fu S, Wang J, et al: De novo centromere formation and centromeric sequence expansion in wheat and its wide hybrids. PLoS Genet 12:e1005997 (2016).

-Han F, Lamb JC, McCaw ME, Gao Z, Zhang B, et al: Meiotic studies on combinations of chromosomes with different sized centromeres in maize. Front Plant Sci 9:785 (2018).

Henikoff S, Ahmad K, Malik HS: The centromere paradox: stable inheritance with rapidly evolving DNA. Science 293:1098-1102 (2001).
International Wheat Genome Sequencing Consortium (IWGSC): Shifting the limits in wheat research and breeding using a fully annotated reference genome. Science 361:eaar7191 (2018).

Kaszas E, Birchler JA: Misdivision analysis of centromere structure in maize. EMBO J 15:52465255 (1996).

Koo DH, Sehgal SK, Friebe B, Gill BS: Structure and stability of telocentric chromosomes in wheat. PLoS One 10:e137747 (2015).

Liu C, Qi L, Liu W, Zhao W, Wilson J, et al: Development of a set of compensating Triticum aestivum-Dasypyrum villosum Robertsonian translocation lines. Genome 54:836-844 (2011).

Lukaszewski AJ: Reconstruction in wheat of complete chromosomes $1 \mathrm{~B}$ and $1 \mathrm{R}$ from the 1RS.1BL translocation of "Kavkaz" origin. Genome 36:821-824 (1993).

Lukaszewski AJ: Chromatid and chromosome type breakage-fusion-bridge cycles in wheat (Triticum aestivum L.). Genetics 140:10691085 (1995).

Lukaszewski AJ: Further manipulation by centric misdivision of the 1RS.1BL translocation in wheat. Euphytica 94:257-261 (1997a).

Lukaszewski AJ: Construction of midget chromosomes in wheat. Genome 40:566-569 (1997b).

Lukaszewski AJ: Behavior of centromeres in univalents and centric misdivision in wheat. Cytogenet Genome Res 129:97-109 (2010).

Lukaszewski AJ: Misdivision of centromeres, in Jiang J, Birchler JA (eds): Plant Centromere Biology, pp 111-128 (John Wiley and Sons, Inc., Ames 2013).

Lukaszewski AJ: A set of new 1RS translocations from wheat cv. Amigo in a uniform genetic background. Euphytica 213:214 (2017).

Lukaszewski AJ, Kopecky D, Linc G: Inversions of chromosome arms $4 \mathrm{AL}$ and $2 \mathrm{BS}$ in wheat invert the patterns of chiasma distribution. Chromosoma 121:201-208 (2012).
- Massoudi-Nejad A, Nasuda S, McIntosh RA, Endo TR: Transfer of rye chromosome segments to wheat by gametocidal system. Chromosome Res 10:349-357 (2002).

Paliulis LV, Nicklas RB: Kinetochore rearrangement in meiosis II requires attachment to the spindle. Chromosoma 113:440-446 (2005).

- Phelps-Durr TL, Birchler JA: An asymptotic determination of minimum centromere size for the maize B chromosome. Cytogenet Genome Res 106:309-313 (2004).

Sears ER: The behavior of isochromosomes and telocentrics in wheat. Chromosoma 4:535550 (1952).

Sears ER, Sears LMS: The telocentric chromosomes of wheat, in Ramanujam S (ed): Proc 5th Int Wheat Genet Symp, pp 389-407 (The Indian Society of Genetics and Plant Breeding, Indian Agricultural Research Institute, New Delhi 1978).

- Sears LMR: Misdivision of five different 3B monosomes in Chinese Spring wheat. Wheat Inf Service 37:9-13 (1973).

Sebesta EE, Wood EA: Transfer of greenbug resistance from rye to wheat with $\mathrm{X}$-rays, in: Agronomy Abstracts ASA, pp 61-62, Madison (1978).

Wang J, Liu Y, Su H, Guo X, Han F: Centromere structure and function analysis in wheat-rye translocation lines. Plant J 91:199-207 (2017).

-Wyman C, Kanaar R: DNA double-strand break repair: all's well that ends well. Annu Rev Genet 40:363-383 (2006).

Yu HG, Dawe RK: Functional redundancy in the maize meiotic kinetochore. J Cell Biol 151: 131-142 (2000).

Zhang P, Friebe B, Lukaszewski AJ, Gill BS: The centromere structure in Robertsonian wheatrye translocation chromosomes indicates that centric breakage-fusion can occur at different positions within the primary constriction. Chromosoma 110:335-344 (2001).
Misdivision of Telocentrics and Isochromosomes
Cytogenet Genome Res 2019;157:179-188 DOI: 10.1159/000497301 